\title{
Resultados a longo prazo de osteotomia varizante femoral distal (OVFD) com cunha de abertura lateral para artrose do compartimento lateral do joelho utilizando navegação computadorizada*
}

\section{Long Term Outcomes of Computer-Navigated Lateral Opening Wedge Distal Femoral Osteotomy for Lateral Compartment Knee Arthrosis}

\author{
Vikram Kandhari ${ }^{1}$ Darshan Angadi ${ }^{10}$ Darli Myat ${ }^{1}$ B Brett Fritsch $^{1}$ () David Parker $^{1}$ \\ Myles Coolican ${ }^{10}$ \\ 1 Departamento de Cirurgia Ortopédica do Joelho, Instituto de \\ Pesquisa Ortopédica de Sydney, Chatswood, Sydney, NSW, Austrália \\ Endereço para correspondência Vikram Kishor Kandhari, MS, 445, \\ Rev Bras Ortop 2022;57(1):96-102. \\ Victoria Avenue, Chatswood, Sydney, NSW, Austrália \\ (e-mail: dr.vikramkandhari@gmail.com).
}

\begin{abstract}
Resumo
Objetivo O objetivo principal do presente estudo foi avaliar os resultados a longo prazo, incluindo a sobrevivência em Osteotomia Varizante Femoral Distal com Cunha de Abertura Lateral (OVFD-CAL) utilizando navegação computadorizada. O objetivo principal do presente estudo foi avaliar os resultados a longo prazo, incluindo a sobrevivência.

Métodos Foi realizada uma análise retrospectiva dos dados coletados prospectivamente de pacientes com artrite do compartimento lateral submetidos a OVFD-CAL por navegação de dezembro de 2006 a novembro de 2012. As pontuações International Knee Documentation Committee (IKDC, na sigla em inglês) e Knee Injury and Osteoarthritis Outcome Score (KOOS, na sigla em inglês) foram analisadas para medição de resultados. Conversão para artroplastia durante o acompanhamento foi o ponto final.

Palavras-chave

- osteoartrite

- joelho

- osteotomia

- cirurgia assistida por computador

- sobrevivência

Resultados Um total de 19 OVFD-CAL foram realizados em 17 pacientes com média de idade de 46,6 $\pm 6,5$ anos formaram a coorte do estudo. $O$ alinhamento coronal foi corrigido a partir de uma média de $7,1^{\circ}\left(2-11^{\circ}\right)$ de valgo para uma média de $2,1^{\circ}\left(0,5^{\circ}-\right.$ $3^{\circ}$ ) de varo. As pontuações do IKDC melhoraram de uma média pré-operatória de 39 para 53 no acompanhamento de médio de longo prazo de 9,1 anos. Os escores do KOOS no acompanhamento a longo prazo foram: dor 71 , sintomas 56 , atividades da vida diária 82, esportes e recreação 59, qualidade de vida 43. A sobrevivência do OVFD-
\end{abstract}

\footnotetext{
Trabalho desenvolvido no Departamento de Cirurgia Ortopédica do Joelho, Instituto de Pesquisa Ortopédica de Sydney, Chatswood, Sydney, NSW, Austrália.
}

recebido

17 de Maio de 2020

aceito

06 de Julho de 2020

Publicado on-line

Setembro 30, 2020
DOI https://doi.org/ 10.1055/s-0040-1716765. ISSN 0102-3616.
(C) 2020. Sociedade Brasileira de Ortopedia e Traumatologia. All rights reserved.

This is an open access article published by Thieme under the terms of the Creative Commons Attribution-NonDerivative-NonCommercial-License, permitting copying and reproduction so long as the original work is given appropriate credit. Contents may not be used for commercial purposes, or adapted, remixed, transformed or built upon. (https://creativecommons.org/ licenses/by-nc-nd/4.0/)

Thieme Revinter Publicações Ltda., Rua do Matoso 170, Rio de Janeiro, RJ, CEP 20270-135, Brazil 


\begin{abstract}
Keywords

- osteoarthritis

- knee

- osteotomy

- surgery, computerassisted

- survivorship

Objective The primary aim of the present study was to evaluate the long-term outcomes including survivorship of computer navigated distal femoral lateral opening wedge osteotomy (DFLOWO). The secondary aim was to identify the potential factors that may influence its survivorship.

Methods A retrospective analysis of prospectively collected data for patients with lateral compartment arthritis who underwent navigated DFLOWO from December 2006 to November 2012 was performed. The International Knee Documentation Committee (IKDC) and Knee Injury and Osteoarthritis Outcome Score (KOOS) scores were analyzed for outcome measures. Conversion to arthroplasty during the follow-up was the end point. Results A total of 19 DFLOWOs were performed in 17 patients with a mean age of $46.6 \pm 6.5$ years formed the study cohort. The coronal alignment was corrected from a mean of $7.1^{\circ}\left(2-11^{\circ}\right)$ valgus to a mean of $2.1^{\circ}\left(0.5^{\circ}-3^{\circ}\right)$ varus. The IKDC scores improved from mean of 39 preoperatively to 53 at the mean long-term follow-up of 9.1 years. The mean KOOS scores at the long-term follow-up were pain 71 , symptoms 56 , activities of daily living 82 , sports and recreation 59 , quality of life 43 . Survivorship of the DFLOWO was $78.9 \%$ at a follow-up of 9.1 years. Presence of $\geq$ grade 2 according to the International Cartilage Repair Society (ICRS) cartilage degeneration in the medial compartment of the knee and $>7^{\circ}$ preoperative valgus deformity were strongly correlated with conversion to total knee arthroplasty (TKA) at the long-term followup $(r=0.66)$.

Conclusions Computer navigated DFLOWO has satisfactory clinical outcomes and $79 \%$ survivorship in long-term follow-up. Presence of ICRS $\geq$ grade 2 degenerative changes in the medial compartment of knee with $>7^{\circ}$ preoperative valgus deformity negatively affects the survivorship of DFLOWO in the long-term follow-up.
\end{abstract}

CAL foi de 78,9\% em um acompanhamento de 9,1 anos. Presença de degeneração da cartilagem segundo a Sociedade Internacional de Reparação de Cartilagem (International Cartilage Repair Society [ICRS, na sigla em inglês]) $\geq$ grau 2 no compartimento medial do joelho e deformidade pré-operatória em valgo $>7^{\circ}$ fortemente correlacionado com a conversão para artroplastia total do joelho (ATJ) no acompanhamento a longo prazo $(r-0,66)$.

Conclusões A OVFD-CAL por navegação computadorizada apresentou resultados clínicos satisfatórios e sobrevida de $79 \%$ no acompanhamento a longo prazo. Presença de alterações degenerativas ICRS $\geq$ grau 2 no compartimento medial do joelho com $>7^{\circ}$ de deformidade pré-operatória em valgo afeta negativamente a sobrevivência da OVFD-CAL no acompanhamento de longo prazo.

\section{Introdução}

Osteotomia do fêmur distal (OVFD) para tratar a degeneração sintomática do joelho é um procedimento bem estabelecido, ${ }^{1}$ com bons resultados relatados na literatura. ${ }^{2}$ Em geral, a osteotomia é baseada em uma técnica de alinhamento com cunha de abertura ${ }^{3-6}$ ou fechamento. ${ }^{7-12}$ Vários parâmetros relacionados ao paciente, incluindo idade, gênero, índice de massa corporal (IMC) e fatores cirúrgicos, como local da osteotomia, "gap" e técnica de fixação, entre outros, demonstraram influenciar o resultado da OVFD. 0 planejamento pré-operatório baseado em imagens é vital para avaliar o tipo e a extensão da correção da deformidade a ser realizada para alcançar o alinhamento ideal. ${ }^{13,14}$
Cirurgia assistida por computador (CAC) tem sido amplamente utilizada como auxílio dinâmico ao alinhamento intraoperatório na artroplastia do joelho, proporcionando bons resultados. ${ }^{15}$ Vários pesquisadores da literatura atual utilizaram a navegação por computador na osteotomia tibial proximal e relataram maior precisão na realização do alinhamento planejado. ${ }^{16-18}$ No entanto, há escassez de estudos relatando os resultados a longo prazo da Osteotomia Varizante Femoral Distal com Cunha de Abertura Lateral (OVFD-CAL) usando a navegação por computador. Através do nosso estudo, pretendemos relatar os resultados a longo prazo e a sobrevivência da OFVD com navegação computadorizada. 


\section{Métodos}

A aprovação do comitê de ética foi obtida para este estudo. Foi dado o devido consentimento para o uso dos dados de todos os pacientes deste estudo. Foi realizada uma análise retrospectiva dos dados coletados prospectivamente de pacientes com artrite do compartimento lateral submetidos a OVFD-CAL utilizando navegação computadorizada de dezembro de 2006 a novembro de 2012. Pacientes consecutivos de artrose do joelho do compartimento lateral com $<55$ anos que não responderam ao tratamento conservador foram incluídos no estudo. Pacientes que apresentaram artrite em estágio terminal com deformidade de flexão fixa de $>15^{\circ},<90^{\circ}$ foram excluídos graus de flexão do joelho ou que não consentiram no procedimento.

Os pacientes incluídos foram operados por um dos três cirurgiões seniores vinculados ao nosso instituto de pesquisa. Os pacientes incluídos foram acompanhados com avaliação clínica e radiológica em intervalos regulares (6 semanas/6 meses/1 ano/5 anos). 0 acompanhamento final dos pacientes incluídos foi realizado por meio de questionário por e-mail das medidas de desfecho relatadas pelo paciente. Conversão em artroplastia uniompartimental do joelho (AUJ) ou artroplastia total do joelho (ATJ) durante o acompanhamento foi o ponto final. Além disso, essas informações foram verificadas no banco de dados do Registro Nacional de Substituição de Articulações da Associação Ortopédica Australiana (AOANJRR, na sigla em inglês). ${ }^{19}$

\section{Técnica Cirúrgica}

A cirurgia foi realizada em decúbito dorsal. Foi feita a preparação e o drapeamento estéril livre do membro inferior. Rastreadores femorais e tibiais foram inseridos para registrar os pontos de referência para medir o alinhamento do membro usando a navegação. Os três cirurgiões usaram dois sistemas de navegação diferentes, incluindo ORTHOSoft (1Zimmer, Warsaw, IN, EUA) e OrthoMap Precision Knee Navigation (Stryker, Kalamazoo, MI, EUA). Primeiro, o centro da cabeça femoral foi registrado. Posteriormente, a artroscopia diagnóstica foi realizada e quaisquer causas intraarticulares de dor no joelho tratáveis por artroscopia foram abordadas. O Grau da Sociedade Internacional de Reparação de Cartilagem (International Cartilage Repair Society [ICRS, na sigla em inglês]) da cartilagem articular do joelho foi documentada para o compartimento medial, lateral, patela e tróclea. O registro dos marcos remanescentes foi concluído, incluindo o centro do fêmur distal e da tíbia proximal, bem como o côndilo femoral e o platô tibial e os maléolos.

Foi realizada uma incisão lateral na pele de 12 a $15 \mathrm{~cm}$ proximalmente a partir do nível da linha lateral da articulação do joelho. A fáscia lata foi incisada imediatamente posterior à banda iliotibial e o vasto lateral foi exposto e elevado anteriormente fora do septo intermuscular lateral. A dissecção subperiosteal do tecido mole do fêmur posterior foi realizada para separar as estruturas neurovasculares posteriores da superfície femoral posterior. Afastadores protetores de tecidos moles foram utilizados para proteger as estruturas neurovasculares e o mecanismo extensor anterior. Utilizando orientação fluoroscópica, passou-se um fio-guia do meio da superfície femoral lateral, proximal ao pólo superior da patela e ligeiramente oblíqua ao plano axial, apontando um pouco distalmente para o tubérculo adutor. Utilizando fluoroscopia e serra sagital, a osteotomia foi iniciada através do osso metafisário da lateral para a medial imediatamente proximal ao fio-guia. Enquanto a serra foi utilizada para osteotomia do fêmur lateral e anterior, um osteótomo agudo foi usado para realizar a osteotomia do córtex posterior. A dobradiça medial do osso intacto foi preservada através do avanço cuidadoso da serra e do osteótomo, mas não além da linha correspondente à face lateral do côndilo femoral medial. Após a osteotomia, o alinhamento foi corrigido para o eixo mecânico neutro sob a orientação da navegação, utilizando um afastador "spreader" laminar para criar um espaço controlado no local da osteotomia, com cuidado para não alterar o fêmur no plano sagital. Uma vez alcançada a correção adequada, o vazio ósseo em forma de cunha foi preenchido com aloenxerto e a osteotomia foi estabilizada com fixação da placa.

No pós-operatório, o membro foi mantido em extensão total em uma faixa de movimento limitada, que foi aberta para permitir uma faixa de $0^{\circ}$ a $30^{\circ}$ no primeiro dia de pósoperatório. A mobilização parcial do suporte de peso foi permitida com suporte - inicialmente um quarto do peso corporal, conforme tolerado pelo paciente e aumentando em um quarto do peso corporal a cada 2 semanas. Em 2 semanas, a cinta foi aberta para permitir uma faixa de $0^{\circ}$ a $90^{\circ}$ graus e a flexão gradual foi permitida com a supervisão de um fisioterapeuta até a marca de 6 semanas, em que a cinta foi descontinuada. $\mathrm{O}$ uso de muletas continua conforme necessário e após 6 semanas o regime de fisioterapia foi atualizado da amplitude do movimento para o fortalecimento do programa de exercícios. Isso foi feito até que o paciente foi capaz de suportar o peso total e andar com apoio.

\section{Medidas e Estatísticas de Resultados}

O Comitê Internacional de Documentação do Joelho (International Knee Documentation Committee [IKDC, na sigla em inglês] $)^{20}$ e o Pontuação de Resultado de Lesão no joelho e de osteoartrite (Knee Injury and Osteoarthritis Outcome Score [KOOS, na siga em inglês) $)^{21}$ foram coletados prospectivamente. A pontuação de IKDC foi obtida no pré-operatório e após 1 ano e, em seguida, no acompanhamento a longo prazo ( $>6$ anos), e os escores do KOOS foram registrados no acompanhamento a longo prazo ( $>6$ anos). Os dados coletados foram registrados na planilha Microsoft Excel (Microsoft Corporation, Redmond, WA, EUA) e analisados usando o software IBM SPSS Statistics for Windows 24 (IBM Corp., Armonk, NY, EUA). Estatísticas descritivas, incluindo média, desvio padrão (DP) e intervalo, foram quantificadas para todos os dados registrados. $\mathrm{O}$ teste $\mathrm{t}$ de Student e a análise de variância (ANOVA) foram utilizados para comparar as médias de 2 ou $>2$ grupos independentes, respectivamente. Análises de regressão linear e múltipla foram utilizadas para identificar os fatores que se correlacionam com a conversão para ATJ. 


\section{Resultados}

Um total de 19 OVFD-CAL foram realizados em 17 pacientes, incluindo 11 homens e 6 mulheres. A média de idade foi de $46,6 \pm 6,5$ (33-53) anos. Um total de 15 das 19 OVFD-CAL (78.9\%) tinham história prévia de meniscectomia lateral parcial. $\mathrm{O}$ alinhamento coronal foi corrigido a partir de uma média de $7,1^{\circ}\left(2-11^{\circ}\right)$ de valgo e para uma média de $2,1^{\circ}\left(0,5^{\circ}-3^{\circ}\right)$ de varo. $O$ alinhamento sagital foi corrigido de uma média de $7,5^{\circ}$ $\left(0-11^{\circ}\right)$ de contratura de flexão para uma média de $1^{\circ}\left(0-3^{\circ}\right)$ de contratura de flexão. Um total de 4 das 19 OVFD-CAL foram convertidos para ATJ. Assim, a sobrevivência do OVFD-CAL navegado em nossa coorte de estudo foi de 78,9\% em um seguimento médio de 9,1 anos (-Fig. 1). As pontuações do IKDC melhoraram da média de 39 no período pré-operatório para 58 no acompanhamento de 1 ano e deterioraram-se para 53 no acompanhamento médio de longo prazo de 9,1 anos. (-Fig. 2). As pontuações do KOOS no acompanhamento a longo prazo foram: dor 71 , sintomas 56 , atividades da vida diária 82 , esportes e recreação 59 , qualidade de vida 43 (-Fig. 3). A amplitude de movimento do joelho para a coorte variou de média de $3^{\circ}$ de contratura em flexão a $124^{\circ}$ de flexão máxima. 0 grau de deformidade pré-operatória em valgo correlacionou-se negativamente com a sobrevivência [Coeficiente de correlação de Pearson [CCP]: $(-0,49) ; p=0,03)]$. Além disso, presença de danos na cartilagem do ICRS de grau $\geq 2$ no compartimento medial do joelho com maior deformidade pré-operatória em valgo $\left(>7^{\circ}\right.$ ) era fortemente correlacionado com a conversão para ATJ no acompanhamento a longo prazo $[r=0,66, p=0,002]$ ( - Tabela 1 ).

Além da conversão para ATJ, 12 intervenções cirúrgicas adicionais foram realizadas na coorte do estudo durante 0 período de acompanhamento. Estes incluíram: i) remoção de "hardware" (9), ii) enxerto ósseo e estabilização (2) para não união e iii) fixação de revisão (1) para fratura peri-implantar secundária por queda aos 6 meses após a cirurgia primária que evoluiu para união satisfatória após intervenção. Um paciente sofreu trombose venosa profunda após o procedimento e foi tratado adequadamente, sem consequências a longo prazo.

\section{Discussão}

Houve um ressurgimento do interesse na OVFD para gerenciar a artrite do joelho do compartimento lateral, especialmente no grupo populacional jovem e ativo. Esse interesse surgiu da improvisação de técnicas cirúrgicas e da disponibilidade de melhores implantes de fixação. Um grande avanço técnico foi o uso da navegação por computador para obter uma correção precisa. Embora a OVFD-CAL tenha ganhado recentemente popularidade em relação à convencional osteotomia femoral distal com cunha de fechamento Medial (OVFD-CFM), ${ }^{1,2}$ há escassez de estudos na literatura atual que relatem os resultados a longo prazo e a sobrevivência após a OVFD-CAL. ${ }^{2}$

Nosso estudo é o primeiro a relatar a sobrevivência a longo prazo de 78,9\% em 9,1 anos de acompanhamento para OVFDCAL navegada por computador. O único outro estudo que relata os resultados clínicos da OVFD navegada por computador é de Saragaglia et al., ${ }^{22}$ o qual incluiu pacientes submetidos a OVFD-CAL ou OVFD-CFM e relatou os resultados clínicos com uma média de acompanhamento de médio prazo de 50,9 meses. No último acompanhamento, nenhum dos pacientes incluídos foi convertido em ATJ. Um estudo recentemente publicado por Shivji et al. ${ }^{23}$ descreveu os resultados clínicos de pacientes de coorte grande de um

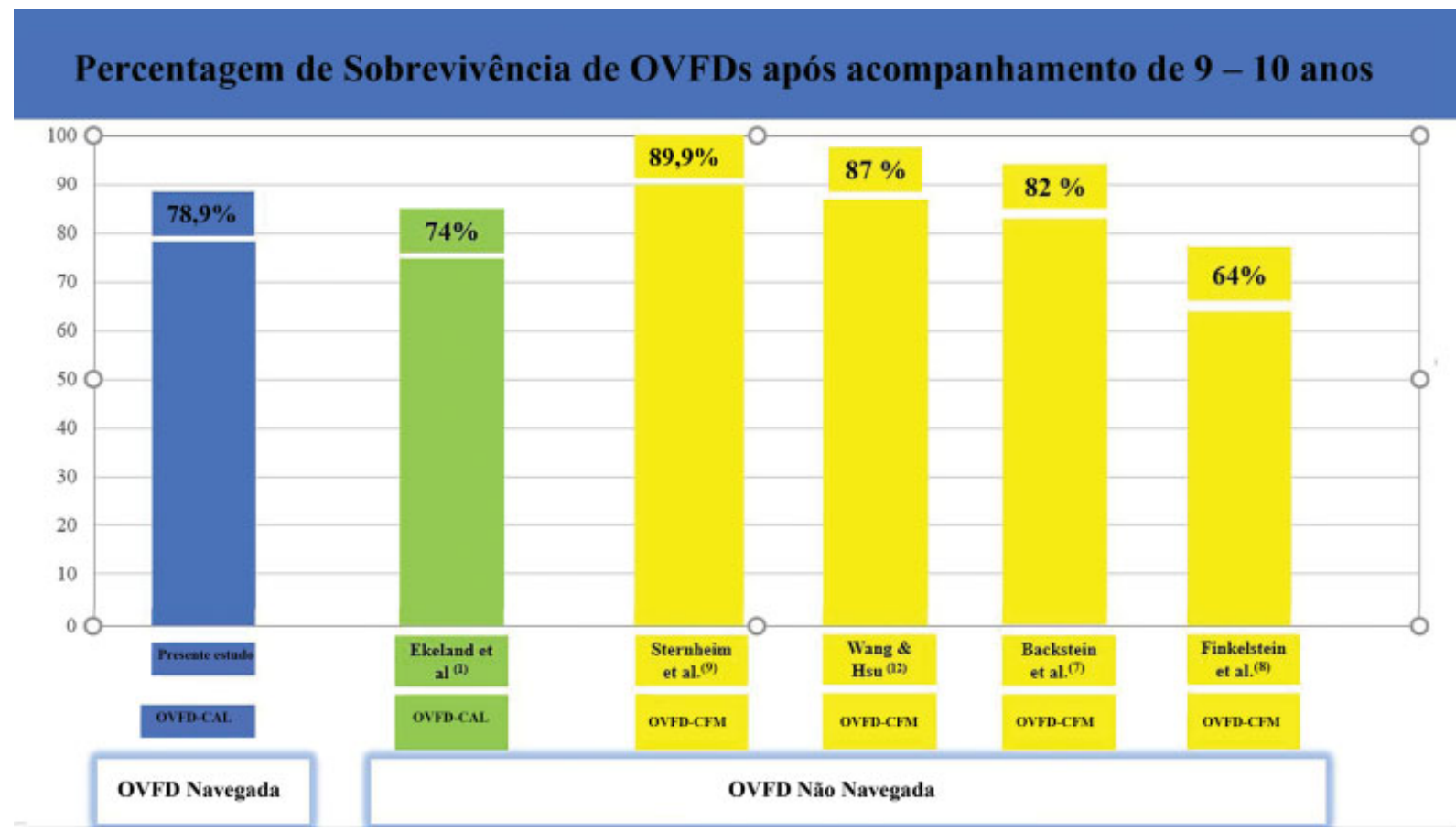

Fig. 1 Sobrevivência comparativa a longo prazo de OVFDs. Legenda: A imagem é um gráfico de barras comparativo da sobrevivência a longo prazo de DFOs realizada para artrose do joelho no compartimento lateral. [OVFD = osteotomia varizante femoral distal; OVFD-CAL = Osteotomia Varizante Femoral Distal com Cunha de Abertura Lateral; OVFD-CFM = Osteotomia Femoral Distal com Cunha de Fechamento Medial]. 


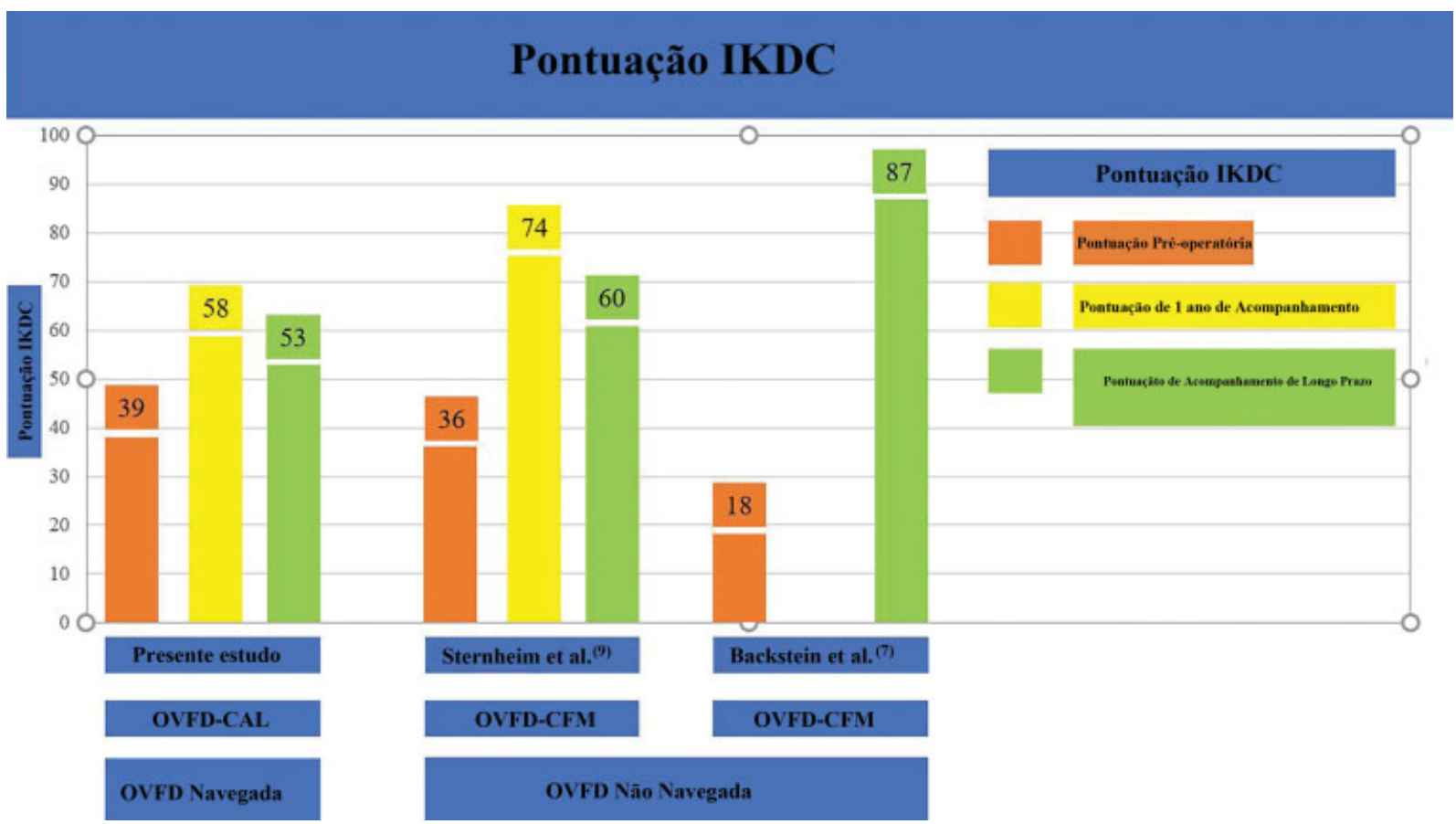

Fig. 2 Gráfico de barras comparativo da pontuação do IKDC. Legenda: A imagem é um gráfico de barras comparativo que descreve a alteração das pontuações do IKDC do período pré-operatório para o acompanhamento a longo prazo [OVFD = osteotomia varizante femoral distal; OVFD$\mathrm{CAL}=$ Osteotomia Varizante Femoral Distal com Cunha de Abertura Lateral; OVFD-CFM = Osteotomia Femoral Distal com Cunha de Fechamento Medial].

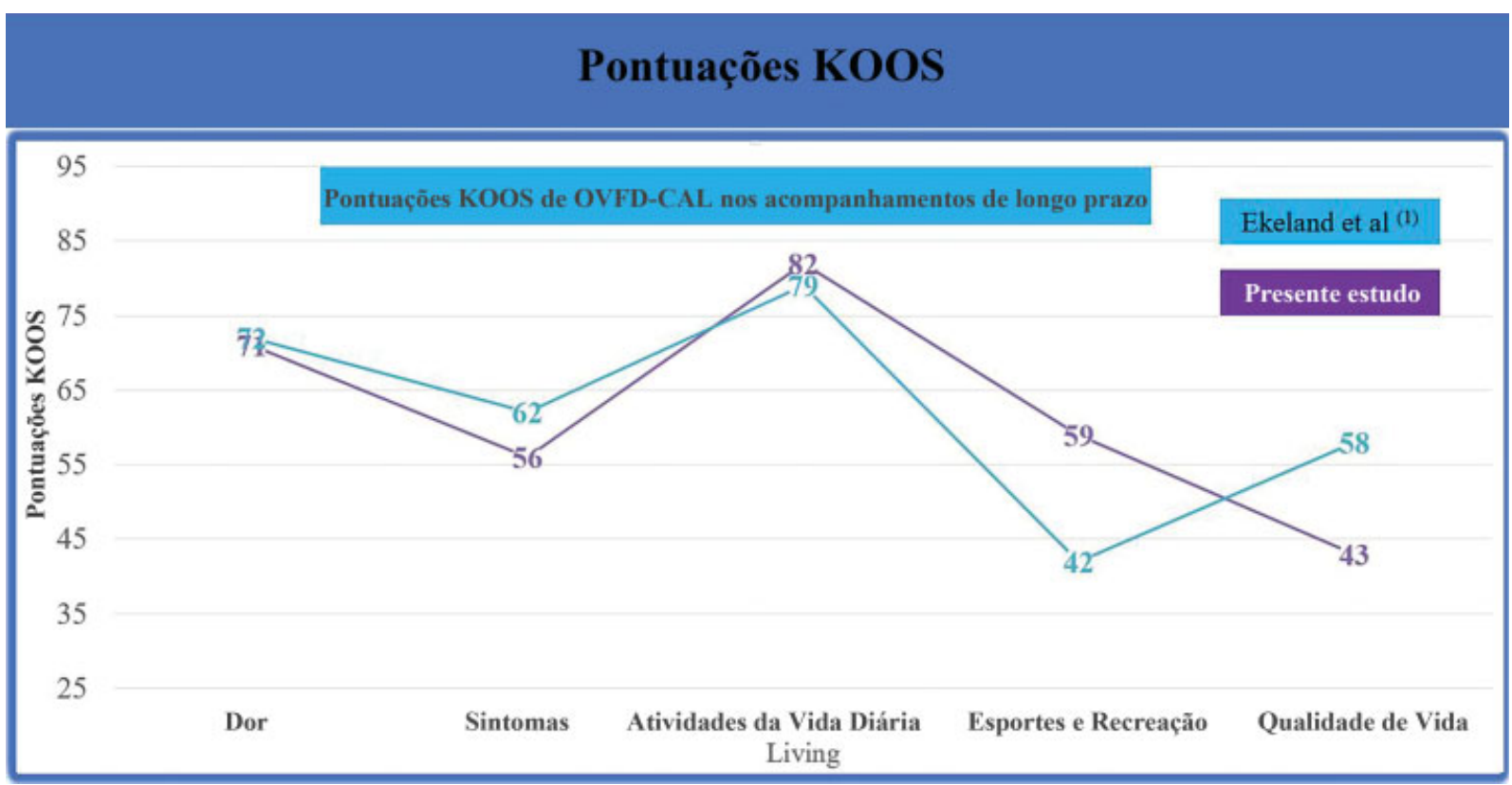

Fig. 3 Pontuação KOOS de OVFD-CALs no acompanhamento a longo prazo. Legenda: A imagem é um diagrama de linhas comparativo que descreve as pontuações KOOS de acompanhamento a longo prazo de OVFD-CAL [OVFD-CAL = Osteotomia Varizante Femoral Distal com Cunha de Abertura Lateral].

único centro submetidos a OVFD-CFM ou OVFD-CAL. Os pacientes incluídos em sua coorte foram submetidos a OVFD após um planejamento digital moderno com o uso de instrumentos cirúrgicos precisos para obter correção e estabilização precisas com a fixação da placa de travamento pré-contornada. Eles relataram uma sobrevida global de $89 \%$ em 10 anos. A sobrevida em longo prazo da osteotomia em nosso estudo é inferior à descrita por Shivji et al. ${ }^{23} \mathrm{~A}$ inclusão de pacientes com apenas OVFD-CAL e não uma coorte mista de OVFD-CAL e OVFD-CFM poderia explicar essa diferença. Além disso, quando comparamos as taxas de sobrevida publicadas de OVFDs com as este estudo, é evidente que a sobrevivência a longo prazo da OVFD-CAL navegada por computador é melhor do que a sobrevivência da OVFD-CAL não navegada, mas inferior à sobrevivência da OVFD-CFM não navegada. (-Fig. 1 ). 
Tabela 1 Fatores que influenciam a sobrevivência de OVFD-CAL

\begin{tabular}{|l|l|l|}
\hline Fatores & CCP & valor-p \\
\hline Gênero & $-0,13$ & 0,61 \\
\hline Idade & 0,28 & 0,25 \\
\hline Valgo Pré-operatório & $-0,49$ & $0,03^{*}$ \\
\hline Grau de Correção & $-0,33$ & 0,17 \\
\hline ICRS Grau de Dano à Cartilagem & & \\
\hline \multicolumn{1}{|c}{ Compartimento medial do joelho } & $-0,28$ & 0,25 \\
\hline $\begin{array}{c}\text { • Compartimento lateral do joelho } \\
\text { - Compartimento femoropatelar }\end{array}$ & 0,05 & 0,84 \\
\hline Meniscectomia lateral anterior & $-0,12$ & 0,62 \\
\hline $\begin{array}{l}> \\
\text { no compré-operatvo Valgo e } \geq \text { grau } 2 \text { ICRS }\end{array}$ & $-0,66$ & $0,002^{*}$ \\
\hline
\end{tabular}

Abreviações: CCP, Coeficiente de correlação de Pearson; ICRS, International Cartilage Repair Society; OVFD-CAL, Osteotomia Varizante Femoral Distal com Cunha de Abertura Lateral.

“*” - Valor de p significante; “““ Correlação negativa.

O uso da artroscopia do joelho no momento da realização da OVFD navegada facilitou o conhecimento do status exato dos danos na cartilagem articular. Pacientes que tinham $\geq$ grau 2 ICRS de danos na cartilagem no compartimento medial do joelho e tinham $>7^{\circ}$ do valgo pré-operatório eram mais propensos a serem convertidos em ATJ $(r=0,66)$. Essas informações importantes podem ajudar na tomada de decisão no pré-operatório e no aconselhamento pós-operatório do paciente. A avaliação do status da cartilagem articular com base na radiografia pode subestimar o dano da cartilagem. Assim, esforços devem ser feitos para obter informações sobre o status da cartilagem do joelho a partir de registros antigos de pacientes, se os pacientes tivessem sido submetidos a artroscopia prévia do joelho. Pacientes jovens com artrose do joelho no compartimento lateral $\geq$ grau 2 ICRS de danos na cartilagem no compartimento medial do joelho e $>7^{\circ}$ de alinhamento do membro de valgo devem ser devidamente informados sobre as altas chances de falha do OVFD-CAL e a necessidade de segunda cirurgia para conversão em ATJ no acompanhamento de longo prazo.

Os escores KOOS de longo prazo dos pacientes submetidos à OVFD-CAL em nosso estudo foram semelhantes aos relatados por Ekeland et al. ${ }^{1}$ em sua série de pacientes submetidos a OVFD-CAL não navegada (-Fig. 3). Quando comparados com os escores publicados de longo prazo do IKDC de pacientes submetidos a OVFD-CFM não navegada, os resultados foram variados ( - Fig. 2). Os escores IKDC de longo prazo dos pacientes de OVFD-CAL navegadas em nossa série foram semelhantes ou marginalmente inferiores aos dos pacientes de OVFD-CFM não navegado; mas nenhuma comparação direta e conclusões puderam ser tiradas, pois esses estudos eram heterogêneos.

Nosso estudo teve certas limitações. Embora nosso estudo tenha sido realizado com os dados de pacientes pertencentes a um instituto, os pacientes incluídos pertenciam a três cirurgiões diferentes que fizeram uso de dois sistemas de navegação diferentes. Embora todos os cirurgiões fossem cirurgiões de joelho especialistas na área e fizessem uso de técnica cirúrgica semelhante, houve diferenças no tipo de fixação e no enxerto ósseo usado. Além disso, o número total de OVFD-CALs incluídas no estudo foi pequeno quando comparado com outras séries publicadas.

\section{Conclusões}

AOVFD-CAL navegada por computador tem resultados clínicos satisfatórios e sobrevida de $79 \%$ em acompanhamento a longo prazo. Presença de alterações degenerativas ICRS $\geq$ grau 2 no compartimento medial do joelho com $>7^{\circ}$ de deformidade pré-operatória em valgo afeta negativamente a sobrevivência da OVFD-CAL no acompanhamento de longo prazo.

Contribuição dos Autores

Todos os autores contribuíram para a formulação do protocolo de ideia e estudo, coleta de dados, análise estatística, análise dos resultados e redação do manuscrito.

\section{Abreviações}

Osteotomia do Fêmur Distal (OVFD), Osteotomia Varizante Femoral Distal com Cunha de Abertura Lateral (OVFD-CAL), Osteotomia Femoral Distal com Cunha de Fechamento Medial (OVFD-CFM), Índice de Massa Corporal (IMC), Comitê Internacional de Documentação do Joelho (IKDC), Pontuação no Resultado da Osteoartrite e Lesão no Joelho (KOOS), Artroplastia Total do Joelho (ATJ), Artroplastia Unicompartimental de Joelho (AUJ), Sociedade Internacional de Reparação de Cartilagem (ICRS), Cirurgia Assistida por Computador (CAC).

\section{Conflitos de Interesse}

Dr. Fritsch relata ser acionista da 360 Knee Systems, Trium e Optimised ortho. Ele fez um trabalho de consultoria para Arthrex, Global Orthopaedics, Omni e fez apresentações pagas para Arthrex, Global Orthopaedics e Omni. Ele recebe apoio institucional de Zimmer, Smith and Nephew, Global Orthopaedics e Arthrex. Acionista da 360 Knee Systems, Trium e Optimised ortho; trabalho de consultoria para 360 Knee Systems, Global Orthopaedics, Omni, Arthrex, Amplitude, Optimized Ortho, Smith and Nephew, Medacta; suporte institucional para Zimmer, Smith and Nephew, Global Orthopaedics, Surgical specialties, Arthrex e Friends of Mater (compra de equipamentos). Dr. Coolican recebeu royalties da Smith and Nephew e trabalha com consultoria para a Smith and Nephew. Ele fez apresentações pagas para a Smith and Nephew, J\&J DePuy, Medacta e recebe apoio institucional da Smith and Nephew e da Corin.

\section{Referências}

1 Ekeland A, Nerhus TK, Dimmen S, Heir S. Good functional results of distal femoral opening-wedge osteotomy of knees with lateral osteoarthritis. Knee Surg Sports Traumatol Arthrosc 2016;24(05): 1702-1709 
2 Chahla J, Mitchell JJ, Liechti DJ, et al. Opening-and closing-wedge distal femoral osteotomy: a systematic review of outcomes for isolated lateral compartment osteoarthritis. Orthop J Sports Med 2016;4(06):2325967116649901

3 Dewilde TR, Dauw J, Vandenneucker H, Bellemans J. Opening wedge distal femoral varus osteotomy using the Puddu plate and calcium phosphate bone cement. Knee Surg Sports Traumatol Arthrosc 2013;21(01):249-254

4 Jacobi M, Wahl P, Bouaicha S, Jakob RP, Gautier E. Distal femoral varus osteotomy: problems associated with the lateral openwedge technique. Arch Orthop Trauma Surg 2011;131(06): 725-728

5 Thein R, Bronak S, Thein R, Haviv B. Distal femoral osteotomy for valgus arthritic knees. J Orthop Sci 2012;17(06):745-749

6 Zarrouk A, Bouzidi R, Karray B, Kammoun S, Mourali S, Kooli M. Distal femoral varus osteotomy outcome: Is associated femoropatellar osteoarthritis consequential? Orthop Traumatol Surg Res 2010;96(06):632-636

7 Backstein D, Morag G, Hanna S, Safir O, Gross A. Long-term followup of distal femoral varus osteotomy of the knee. J Arthroplasty 2007;22(04, Suppl 1):2-6

8 Finkelstein JA, Gross AE, Davis A. Varus osteotomy of the distal part of the femur. A survivorship analysis. J Bone Joint Surg Am 1996;78(09):1348-1352

9 Sternheim A, Garbedian S, Backstein D. Distal femoral varus osteotomy: unloading the lateral compartment: long-term follow-up of 45 medial closing wedge osteotomies. Orthopedics 2011;34(09):e488-e490

10 Kosashvili Y, Safir O, Gross A, Morag G, Lakstein D, Backstein D. Distal femoral varus osteotomy for lateral osteoarthritis of the knee: a minimum ten-year follow-up. Int Orthop 2010;34(02): 249-254

11 Forkel P, Achtnich A, Metzlaff S, Zantop T, Petersen W. Midterm results following medial closed wedge distal femoral osteotomy stabilized with a locking internal fixation device. Knee Surg Sports Traumatol Arthrosc 2015;23(07):2061-2067

12 Wang JW, Hsu CC. Distal femoral varus osteotomy for osteoarthritis of the knee. J Bone Joint Surg Am 2005;87(01): $127-133$
13 Paccola CAJ. Pre-operative planning and surgical technique of the open wedge supracondylar osteotomy for correction of valgus knee and fixation with a fixed-angle implant. Rev Bras Ortop 2015;45(06):627-635

14 Elattar O, Swarup I, Lam A, Nguyen J, Fragomen A, Rozbruch SR. Open wedge distal femoral osteotomy: accuracy of correction and patient outcomes. HSS J 2017;13(02):128-135

15 Lee SH, Song EK, Seon JK. Navigation system provides better functional outcomes of total knee arthroplasty. Form J Musculoskel Dis 2016;7(01):8-16

16 Reising K, Strohm PC, Hauschild O, et al. Computer-assisted navigation for the intraoperative assessment of lower limb alignment in high tibial osteotomy can avoid outliers compared with the conventional technique. Knee Surg Sports Traumatol Arthrosc 2013;21(01):181-188

17 Akamatsu Y, Mitsugi N, Mochida Y, et al. Navigated opening wedge high tibial osteotomy improves intraoperative correction angle compared with conventional method. Knee Surg Sports Traumatol Arthrosc 2012;20(03):586-593

18 Ribeiro CH, Severino NR, Moraes de Barros Fucs PM. Opening wedge high tibial osteotomy: navigation system compared to the conventional technique in a controlled clinical study. Int Orthop 2014;38(08):1627-1631

19 The Australian Orthopaedic Association National Joint Replacement Registry. Ad Hoc Report. Adelaide, Australia: AOA; 2019

20 Irrgang JJ, Anderson AF, Boland AL, et al. Development and validation of the international knee documentation committee subjective knee form. Am J Sports Med 2001;29(05):600-613

21 Roos EM, Roos HP, Lohmander LS, Ekdahl C, Beynnon BD. Knee Injury and Osteoarthritis Outcome Score (KOOS)-development of a self-administered outcome measure. J Orthop Sports Phys Ther 1998;28(02):88-96

22 Saragaglia D, Chedal-Bornu B. Computer-assisted osteotomy for valgus knees: medium-term results of 29 cases. Orthop Traumatol Surg Res 2014;100(05):527-530

23 Shivji FS, Foster A, Risebury MJ, Wilson AJ, Yasen SK. Ten-year survival rate of $89 \%$ after distal femoral osteotomy surgery for lateral compartment osteoarthritis of the knee. Knee Surg Sports Traumatol Arthrosc 2020. Doi: 10.1007/s00167-020-05988-5 